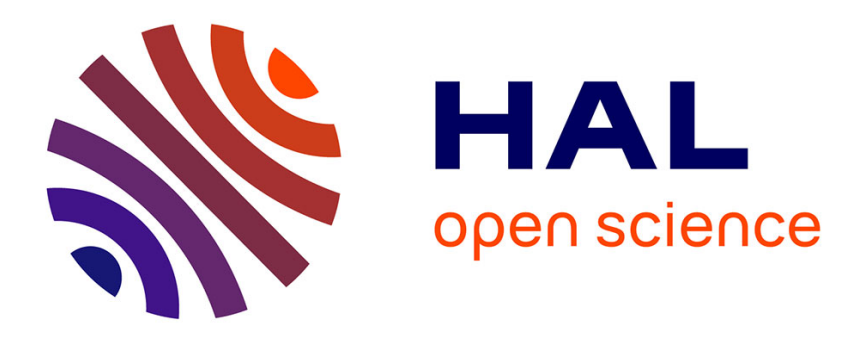

\title{
Mueller matrix interpolation in polarization optics
}

Vincent Devlaminck

\section{To cite this version:}

Vincent Devlaminck. Mueller matrix interpolation in polarization optics. Journal of the Optical Society of America. A Optics, Image Science, and Vision, 2010, 27 (7), pp.1529-1534. hal-00648831

\section{HAL Id: hal-00648831 https://hal.science/hal-00648831}

Submitted on 9 Mar 2012

HAL is a multi-disciplinary open access archive for the deposit and dissemination of scientific research documents, whether they are published or not. The documents may come from teaching and research institutions in France or abroad, or from public or private research centers.
L'archive ouverte pluridisciplinaire HAL, est destinée au dépôt et à la diffusion de documents scientifiques de niveau recherche, publiés ou non, émanant des établissements d'enseignement et de recherche français ou étrangers, des laboratoires publics ou privés. 


\title{
Mueller Matrix interpolation in polarization optics
}

\author{
V. Devlaminck \\ LAGIS - FRE CNRS 3303 \\ Université Lille 1, Sciences et Technologies - 59655 France \\ e.mail : vincent.devlaminck@univ-lille1.fr
}

\section{1 - Introduction}

Mueller matrix as linear mapping between the input and output Stokes vectors of light interacting with media, is a very powerful tool for polarimetric characterization of linear media. The measurement of the Mueller matrix entries attached to each pixel in an image is named Mueller imaging. The development of optical devices and systems [1], calibration procedures [2] and measurement inversion methods [3][4] produced Mueller imaging systems with low noise measurement where the physical constraints on Mueller matrices are taken into account. These works contributed to popularize this imaging approach and examples of Mueller matrix imaging can be found for characterizing biological objects [5] or scattering media [6], with applications in dermatology [7], ophthalmology [8] or physics [9][10] for instance.

Since polarimetric information is now available under image format, there is a growing need to understand the underlying physical model associated with the classical image processing tools applied on Mueller imaging.

In this paper, we address the basic question of the physical meaning of Mueller matrix average. Though the mean value of two Mueller matrices is one of the most elementary operations, the comprehension of the physical meaning and related properties of this operation is of great importance since most of the image processing tools involve averaging procedures. Computing the mean value of two Mueller matrices can be reformulated as the more general question of interpolation over the space of Mueller matrices since the mean value is just one particular point of the path interpolating two points of the space. A well posed approach to define this path of interpolation between two points of a space, is the notion of geodesic curve. A curve is called a geodesic [11] for an associated distance defined on the space, if the length of the curve is exactly the distance between its end points. This definition implies that it is also the shortest curve between any two of its points which obviously is a desirable property for interpolation curves.

But it is clear that we have as many solutions as we have distance definitions on the space. The question of the physical meaning of the averaging operation is then related to the physical meaning of the distance associated to the geodesic curves.

After a reminder of definitions and relations associated with Mueller and coherency matrix, we derive the expressions of two distances in an Euclidean and Riemannian context over the space of coherency matrices. The associated interpolation procedures are depicted with the underlying physical model. Eventually, properties of both the solutions are presented.

\section{2 - Distances on Mueller and coherency matrix space.}

\subsection{Mueller and coherency matrix definition.}

Following Kim et al. [12], we define a Mueller matrix $\mathbf{M}$ as a convex sum of so-called Mueller-Jones matrices also named pure Mueller matrices or nondepolarizing Mueller matrices These matrices are obtained by writing the equation $\mathbf{E}^{\prime}=\mathbf{J E}$, mapping an input electric field vector $\mathbf{E}$ into an output electric field vector $\mathbf{E}^{\prime}$ by means of the (generally complex) $2 \times 2$ Jones matrix $\mathbf{J}$, in terms of a relation between the corresponding 
vectors of Stokes parameters. There exist complete characterizations of such a class of real $4 \times 4$ matrices. For instance, Cloude [13] proved that a real $4 \times 4$ matrix $\mathbf{M}$ is a Mueller matrix with respect to the previous definition if and only if the (complex hermitian) $4 \times 4$ coherency matrix $\mathbf{C}$ constructed from $\mathbf{M}$ by linear operations has only nonnegative eigenvalues.

The underlying physical model associated to this definition, is obtained by considering the optical system with Mueller matrix $\mathbf{M}$, as an ensemble. Each realization " $k$ " is characterized by a Jones matrix $\mathbf{J}(\mathrm{k})$ occurring with a probability $\mathrm{p}(\mathrm{k})$.It is also possible to consider the optical system as composed of a set of parallel elements characterized by a deterministic Jones matrix $\mathbf{J}(\mathrm{k})$ in such a manner that the light beam is shared among these elements according to a ratio $\mathrm{p}(\mathrm{k})=\mathrm{I}(\mathrm{k}) / \mathrm{I}$ where $\mathrm{I}(\mathrm{k})$ is the intensity of the portion of light interacting with element $\mathrm{k}$ and $\mathrm{I}$ is the intensity of the whole beam [14].

A straightforward application of the physical model considering the system as an ensemble, gives [12] the following relations:

$$
\mathbf{M}=\left(\mathrm{m}_{\mathrm{ij}}\right)=\Lambda^{\dagger} \mathbf{F} \Lambda \text { and } \mathbf{F}=\Lambda \mathbf{M} \Lambda^{\dagger}
$$

where $\mathbf{F}$ is defined as the average value of Kronecker product of the $\mathbf{J}$ matrix and its conjugate $\mathbf{J}^{*}$ ( $\dagger$ and $*$ stand for a Hermitian and complex conjugate respectively).

$$
\begin{aligned}
& \Lambda=\frac{1}{\sqrt{2}}\left[\begin{array}{cccc}
1 & 1 & 0 & 0 \\
0 & 0 & 1 & -\mathrm{i} \\
0 & 0 & 1 & \mathrm{i} \\
1 & -1 & 0 & 0
\end{array}\right] \quad \mathbf{F}=\left\langle\mathbf{J} \otimes \mathbf{J}^{*}\right\rangle_{\mathrm{e}}=\left[\begin{array}{llll}
\left\langle\mathrm{j}_{0} \mathrm{j}_{0}^{*}\right\rangle_{\mathrm{e}} & \left\langle\mathrm{j}_{0} \mathrm{j}_{1}^{*}\right\rangle_{\mathrm{e}} & \left\langle\mathrm{j}_{1} \mathrm{j}_{0}^{*}\right\rangle_{\mathrm{e}} & \left\langle\mathrm{j}_{1} \mathrm{j}_{1}^{*}\right\rangle_{\mathrm{e}} \\
\left\langle\mathrm{j}_{0} \mathrm{j}_{2}^{*}\right\rangle_{\mathrm{e}} & \left\langle\mathrm{j}_{0} \mathrm{j}_{3}^{*}\right\rangle_{\mathrm{e}} & \left\langle\mathrm{j}_{1} \mathrm{j}_{2}^{*}\right\rangle_{\mathrm{e}} & \left\langle\mathrm{j}_{1} \mathrm{j}_{3}^{*}\right\rangle_{\mathrm{e}} \\
\left\langle\mathrm{j}_{2} \mathrm{j}_{0}^{*}\right\rangle_{\mathrm{e}} & \left\langle\mathrm{j}_{2} \mathrm{j}_{1}^{*}\right\rangle_{\mathrm{e}} & \left\langle\mathrm{j}_{3} \mathrm{j}_{0}^{*}\right\rangle_{\mathrm{e}} & \left\langle\mathrm{j}_{3} \mathrm{j}_{1}^{*}\right\rangle_{\mathrm{e}} \\
\left\langle\mathrm{j}_{2} \mathrm{j}_{2}^{*}\right\rangle_{\mathrm{e}} & \left\langle\mathrm{j}_{2} \mathrm{j}_{3}^{*}\right\rangle_{\mathrm{e}} & \left\langle\mathrm{j}_{3} \mathrm{j}_{2}\right\rangle_{\mathrm{e}} & \left\langle\mathrm{j}_{3} \mathrm{j}_{3}^{*}\right\rangle_{\mathrm{e}}
\end{array}\right] \\
& \mathbf{J}=\left[\begin{array}{lll}
\mathrm{j}_{0} & \mathrm{j}_{1} \\
\mathrm{j}_{2} & \mathrm{j}_{3}
\end{array}\right]
\end{aligned}
$$

Although Mueller matrix has not any particular symmetry property, it is possible to extract from it an Hermitian matrix $\mathbf{H}$ by doing a partial exchange of the $\mathbf{F}$ rows. This mapping $\mathbf{H}=\operatorname{Per}(\mathbf{F})$, (see Appendix A for the definition of this mapping ) transforming $\mathbf{F}$ matrix into $\mathbf{H}$ is related to the Mueller matrix elements $\mathrm{m}_{\mathrm{ij}}$ and the Pauli matrices $\left(\boldsymbol{\sigma}_{\mathrm{i}}\right)$ by:

$$
\mathbf{H}=\sum_{\mathrm{ij}} \mathrm{m}_{\mathrm{ij}}\left(\sigma_{\mathrm{i}} \otimes \sigma_{\mathrm{j}}^{*}\right)=\operatorname{Per}(\mathbf{F})=\operatorname{Per}\left(\Lambda \mathbf{M} \Lambda^{\dagger}\right)
$$

The corresponding mapping from Mueller matrix space to Hermitian matrix space, is noted by : $\varphi(\mathbf{M})=\mathbf{H}$. Its inverse is noted $\psi(\mathbf{H})=\mathbf{M}=\Lambda^{\dagger} \operatorname{Per}(\mathbf{H}) \boldsymbol{\Lambda}$ since $(\text { Per })^{-1}=$ Per.

The coherency matrix $\mathbf{C}$ is related to $\mathbf{H}$ by:

$$
\mathbf{C}=\Lambda^{\dagger} \mathbf{H} \Lambda
$$

In fact, as demonstrated by Aiello et al. [15], there are infinitely many Hermitian matrices generated by $\mathbf{M}$ which differ from $\mathbf{H}$ by an unitary transformation. Each of these Hermitian matrices is related to the coefficients of the decomposition of matrix $\mathbf{F}$ on a specific basis of $\mathbb{C}^{4 \times 4}$. $\mathbf{H}$ is related to the standard basis for instance and $\mathbf{C}$ to the basis derived from the Kronecker product of Pauli matrices.

\subsection{Distances on Mueller or coherency matrix space.}

Before defining a distance on a space, the first step is to define the space under analysis. It is worth 
noticing that many experimental coherency matrices of media exhibited in the literature are positive definite matrices (their four eigenvalues are strictly positive).Under this hypothesis, the set of coherency matrices may be restricted to $H P D(4)$, the manifold of Hermitian Positive Definite matrices of dimension 4. It is clear that we don't take into account the Mueller matrices corresponding to a singular coherency matrix. The expression of coherency matrix in terms of the statistical parameters of Eq. (2) shows that its eigenvalues are related to the correlation between the Jones matrices coefficients. In polarization optics, these properties are depicted using the notion of structure polarimetric purity and parameters like degree of purity or indices of purity [16][17], giving a measurement of this purity can be found in the literature. Mueller imaging of natural scene (this means for classical material media constituting the most frequently imaged objects) often leads to experimental matrices with low purity indices. It may become from the complexity of the natural media themselves but also from the accuracy of classical Mueller imaging systems where the temporal and spatial resolution of the measurement may be not precise enough.

From the previous relations between Mueller and coherency matrix, it is clear that we can define distances on either one or the other space. As there are infinitely many Hermitian matrices generated by a Mueller matrix which only differ by an unitary transformation, we can expect as a desirable property of a distance on coherency matrix space to be invariant by an unitary transformation applied on coherency matrices. The choice of the basis of $\mathbb{C}^{4 \times 4}$ used to decompose the matrix $\mathbf{F}$ on, has no physical meaning on polarimetric properties and must not influence the result of operations like average or interpolation. These points being noticed, we now analyse two examples of such distances and the corresponding interpolation procedures.

\subsubsection{Euclidean distance}

Using the classical Euclidean distance on the space of Mueller matrices, is a first solution to interpolate Mueller matrices. Since the Mueller matrix space forms a convex set (a Mueller matrix is a positive summation of one to four Mueller-Jones matrices [13]), the summation of Mueller matrices is always a Mueller one. The Euclidean distance between $\mathrm{M}_{1}$ and $\mathrm{M}_{2}$ matrices is defined by:

$$
\mathrm{d}_{\mathrm{E}}\left(\mathbf{M}_{1}, \mathbf{M}_{2}\right)=\left\|\mathbf{M}_{1}-\mathbf{M}_{2}\right\|=\left[\operatorname{Tr}\left[\left(\mathbf{M}_{1}-\mathbf{M}_{2}\right)\left(\mathbf{M}_{1}-\mathbf{M}_{2}\right)^{\dagger}\right]\right]^{1 / 2}
$$

where $\operatorname{Tr}(\mathbf{A})$ denotes the trace of matrix $\mathbf{A}$.

The straight lines are the geodesic curves in Euclidean space. Thus, the linear interpolation between two elements $\mathbf{M}_{1}$ and $\mathbf{M}_{2}$ of Mueller matrix space, is the straight line between both the points. Assuming normalized weights (sum of the weights equals 1), interpolation is a weighted mean following the relation:

$$
\mathbf{M}(\mathrm{t})=(1-t) \mathbf{M}_{1}+t \mathbf{M}_{2} \quad \text { with } 0 \leq t \leq 1
$$

It is worth noticing that computing first the interpolation curve on the $H P D(4)$ matrix space, is also possible:

$$
\begin{aligned}
\mathbf{H}(t) & \left.=\operatorname{Per}\left(\Lambda \mathbf{M}(\mathrm{t}) \Lambda^{\dagger}\right)=\operatorname{Per}\left[\Lambda\left((1-t) \mathbf{M}_{1}+t \mathbf{M}_{2}\right) \Lambda^{\dagger}\right)\right] \\
& =(1-t) \operatorname{Per}\left[\Lambda \mathbf{M}_{1} \Lambda^{\dagger}\right]+t \operatorname{Per}\left[\Lambda \mathbf{M}_{2} \Lambda^{\dagger}\right] \\
& =(1-t) \mathbf{H}_{1}+t \mathbf{H}_{2}
\end{aligned}
$$

Then the $\varphi$ mapping defined by Eq. (3), gives the corresponding Mueller matrix $\mathrm{M}(t)$ of Eq. (6).

A straightforward application of Eq. (4) shows that this result does not depend on the specific basis 
of $\mathbb{C}^{4 \times 4}$ used to generate the $H P D(4)$ matrices from the corresponding $\mathbf{F}$ matrices.

\subsubsection{Log-Euclidean distance}

From algebraic properties and differential geometry properties of $H P D(4)$ when considering $H P D(4)$ matrices as a smooth manifold, it is also possible to define a Riemannian framework on this set of matrices. This is the way used by Arsigny et al. [18] to define a distance on Symmetric Positive Definite matrices termed Log-Euclidean distance ( $L E$ distance). The definition and the properties of this distance can easily be extended to $H P D(4)$ matrices. The reader is referred to [18] for the demonstration of these properties with Symmetric Positive Definite matrices. Proving that these properties are well adapted to Mueller matrix interpolation and deriving the underlying physical model, will be done below.

For two $H P D(4)$ matrices, the $L E$ distance is defined by:

$$
\mathrm{d}_{\mathrm{LE}}\left(\mathbf{H}_{1}, \mathbf{H}_{2}\right)=\left\|\log \left(\mathbf{H}_{1}\right)-\log \left(\mathbf{H}_{2}\right)\right\|=\left[\operatorname{Tr}\left[\left(\log \left(\mathbf{H}_{1}\right)-\log \left(\mathbf{H}_{2}\right)\right)^{2}\right]\right]^{1 / 2}
$$

where $\operatorname{Tr}(\mathbf{A})$ denotes the trace of matrix $\mathbf{A}$.

Eq. (8) shows $L E$ distance has an Euclidean distance on matrix logarithms. It is worth noticing that defining such a distance on the set of Mueller matrices, is not possible. The existence of matrix logarithm is not guaranteed with this set of matrices.

This distance is invariant by similarity (isometry plus scaling). Thus, $\mathrm{d}\left(\mathbf{C}_{1}, \mathbf{C}_{2}\right)=\mathrm{d}\left(\mathbf{H}_{1}, \mathbf{H}_{2}\right)$ for two couples of $H P D(4)$ matrices $\mathrm{C}_{\mathrm{k}}$ and $\mathrm{H}_{\mathrm{k}}$ generated by $\mathbf{M}_{\mathrm{k}}$ (with $\mathrm{k}=1,2$ ) which differ by an unitary transformation $\mathrm{U}$. This means $\mathrm{C}_{1}=\mathrm{U}^{\dagger} \mathrm{H}_{1} \mathrm{U} \mathrm{C}_{2}=\mathrm{U}^{\dagger} \mathrm{H}_{2} \mathrm{U}$. Consequently, the $L E$ distance (as the Euclidean one) does not depend on the specific basis of $\mathbb{C}^{4 \times 4}$ used to generate the $H P D(4)$ matrices from the decomposition of matrix $\mathbf{F}$. With scaling invariance property, a normalization of Mueller matrices by the coefficient $\mathrm{m}_{00}$ of one of them for instance, does not affect the interpolation procedure.

Following the definition of the $L E$ distance, the geodesic curve or interpolation curve between $\mathbf{H}_{1}$ and $\mathbf{H}_{2}$, two elements of $H P D(4)$, is given by:

$$
\mathbf{H}(t)=\exp \left(\left[(1-t) \log \left(\mathbf{H}_{1}\right)+t \log \left(\mathbf{H}_{2}\right)\right]\right)
$$

The corresponding interpolation of Mueller matrices is given by using Eq. (3) but it is worth noticing that if $\mathbf{H}_{1}=\varphi\left(\mathbf{M}_{1}\right)$ and $\mathbf{H}_{2}=\varphi\left(\mathbf{M}_{2}\right)$, computing the $\mathbf{M}(t)$ interpolation curve as a weighted mean of $\mathbf{M}_{1}$ and $\mathbf{M}_{2}$, is impossible.

\section{3 - Physical significance and properties of interpolation processes.}

\subsection{Euclidean interpolation}

The physical meaning of the first interpolation technique based on the Euclidean distance, is obvious. The interpolated matrix $\mathbf{M}(t)$ of Eq. (6) may be considered as the Mueller matrix of an optical system exhibiting spatial heterogeneity over the area illuminated by the incident light. It is composed of two parallel elements characterized by a Mueller matrix $\mathbf{M}_{1}$ and $\mathbf{M}_{2}$. The light beam is shared among these elements according to a ratio (1-t) and $t$ respectively and the emerging light is consequently composed of both these incoherent contributions. The parameter $t$ may be considered as a quantity homogeneous to a surface. Fig.1-(a) illustrates this interpolating process with different values of the parameter. 


\subsection{Log-Euclidean interpolation}

Addressing the question of the physical meaning of this latter process of interpolation, may be founded on a very similar approach to the layered-medium interpretation proposed by Jones in the seventh paper of his series [19].

We first note the following properties:

$\boldsymbol{A}$ - As a consequence of Lie group structure of $H P D(4)$ with the group product defined by $\left(\mathbf{H}_{1} \oplus \mathbf{H}_{2}\right)=\exp \left(\log \left(\mathbf{H}_{1}\right)+\log \left(\mathbf{H}_{2}\right)\right)$, (see [18] for demonstration) the one parameter subgroups of HPD(4) are all of the form:

$$
\mathbf{H}(\tau)=\exp (\tau \mathbf{D})=\mathbf{I}+\tau \mathbf{D}+\frac{\mathbf{1}}{\mathbf{2}}(\tau \mathbf{D})^{2}+\frac{1}{6}(\tau \mathbf{D})^{3}+\cdots
$$

where $\mathbf{D}$ is the infinitesimal generator of the subgroup and $\mathbf{I}$ is the unit matrix.

$\boldsymbol{B}$ - A pure depolarizing material medium has a Mueller matrix $\mathbf{M}_{0}$ with:

$$
\mathbf{M}_{0}=\left[\begin{array}{llll}
1 & 0 & 0 & 0 \\
0 & 0 & 0 & 0 \\
0 & 0 & 0 & 0 \\
0 & 0 & 0 & 0
\end{array}\right]
$$

If a very thin section of a medium is removed from this pure depolarizing element, the resulting Mueller matrix $\mathbf{M}_{1}{ }^{\mathrm{e}}$ differs only slightly from the $\mathbf{M}_{0}$ matrix. The corresponding $H P D$ matrix $\mathbf{H}_{1}{ }^{\mathrm{e}}$ differs only slightly from the unit matrix since from Eq. (3), $\varphi\left(\mathbf{M}_{0}\right)=\mathbf{I}$.

Let the corresponding thickness and generator named $\tau_{1}$ and $\mathbf{D}_{1}$ respectively. From Eq.( 10), this $H P D$ matrix may be written :

$$
\mathbf{H}_{1}^{\mathrm{e}}=\exp \left(\tau_{1} \mathbf{D}_{1}\right)=\mathbf{I}+\tau_{1} \mathbf{D}_{1}+O\left(\tau_{1}^{2}\right)
$$

where the term $O\left(\tau_{1}^{2}\right)$ is for the terms of order higher than one. A second very thin section with parameters $\tau_{2}$ and $\mathbf{D}_{2}$ gives another $H P D$ matrix

$$
\mathbf{H}_{2}^{\mathrm{e}}=\exp \left(\tau_{2} \mathbf{D}_{2}\right)=\mathbf{I}+\tau_{2} \mathbf{D}_{2}+O\left(\tau_{2}^{2}\right)
$$

and a corresponding Mueller matrix $\mathbf{M}_{2}{ }^{\mathrm{e}}$.

Let the product $\mathbf{H}_{\mathrm{s}}=\mathbf{H}_{1}{ }^{\mathrm{e}} \cdot \mathbf{H}_{2}{ }^{\mathrm{e}}$ be now considered. From Eqs. (12 -13):

$$
\begin{aligned}
& \mathbf{H}_{\mathrm{s}}=\mathbf{I}+\tau_{1} \mathbf{D}_{1}+\tau_{2} \mathbf{D}_{2}+O\left[\left(\tau_{1}+\tau_{2}\right)^{2}\right] \text { or } \\
& \mathbf{H}_{\mathrm{s}}=\mathbf{I}+\tau\left[(1-t) \mathbf{D}_{1}+t \mathbf{D}_{2}\right]+O\left(\tau^{2}\right) \text { with } t=\frac{\tau_{2}}{\tau} \text { and } \tau=\tau_{1}+\tau_{2}
\end{aligned}
$$

The matrix $\mathbf{M}_{\mathrm{s}}=\psi\left(\mathbf{H}_{\mathrm{s}}\right)$, may be thus considered as the Mueller matrix of a pure depolarizing element where a very thin section of a medium with a thickness $\tau=\tau_{1}+\tau_{2}$, has been removed (see the second part of Eq. (14)). This medium is characterized by an infinitesimal generator $\overline{\mathbf{D}}=(1-t) \cdot \mathbf{D}_{1}+t \cdot \mathbf{D}_{2}$. This is a sandwich of both the previous media with proportions (1-t) and $t$ respectively. It is worth noticing that this result is independent of the order in which both the sections are placed since $\mathbf{H}_{1}{ }^{\mathrm{e}} \cdot \mathbf{H}_{2}{ }^{\mathrm{e}}=\mathbf{H}_{2}{ }^{\mathrm{e}} \cdot \mathbf{H}_{1}{ }^{\mathrm{e}}$ as proved by Eq. (14).

Thus, using a classical approximation of the logarithm near the unity $: \log (\mathbf{I}+\mathbf{B})=\mathbf{B}+O\left(|\mathbf{B}|^{2}\right)$, we have:

$$
\begin{aligned}
\log \left(\mathbf{H}_{\mathrm{s}}\right)=\log \left(\mathbf{H}_{1}^{\mathrm{e}} \mathbf{H}_{2}^{\mathrm{e}}\right) & =\log \left(\mathbf{I}+\tau\left[(1-t) \mathbf{D}_{1}+t \mathbf{D}_{2}\right]+O\left(\tau^{2}\right)\right) \\
& =\tau\left[(1-t) \mathbf{D}_{1}+t \mathbf{D}_{2}\right]+O\left(\tau^{2}\left\|(1-t) \mathbf{D}_{1}+t \mathbf{D}_{2}\right\|^{2}\right)
\end{aligned}
$$


and if $\tau$ approaches zero :

$$
\mathbf{H}_{\mathrm{s}}=\exp \left(\tau\left[(1-t) \mathbf{D}_{1}+t \mathbf{D}_{2}\right]+O\left(\tau^{2}\left\|(1-t) \mathbf{D}_{1}+t \mathbf{D}_{2}\right\|^{2}\right)\right)
$$

Removing a medium of thickness $z$ from a pure depolarizing element of thickness $L$, is the last step. This medium may be considered as a sandwich composed by $q$ elements of the previous nature. Since $q \tau=z$, we have from Eq. (16):

$$
\begin{aligned}
\left(\mathbf{H}_{\mathrm{s}}\right)^{q}=\left(\mathbf{H}_{1}^{\mathrm{e}} \mathbf{H}_{2}^{\mathrm{e}}\right)^{q} & =\exp \left(q \tau\left[(1-t) \mathbf{D}_{1}+t \mathbf{D}_{2}\right]+O\left(q \tau^{2}\left[(1-t) \mathbf{D}_{1}+t \mathbf{D}_{2}\right]^{2}\right)\right) \\
& =\exp \left(z\left[(1-t) \mathbf{D}_{1}+t \mathbf{D}_{2}\right]+O\left(z \tau\left[(1-t) \mathbf{D}_{1}+t \mathbf{D}_{2}\right]^{2}\right)\right)
\end{aligned}
$$

Thus when $\tau$ approaches zero and $q$ becomes infinite since $z$ is constant:

$$
\mathbf{H}(\mathrm{z}, \mathrm{t})=\lim _{\mathrm{q} \rightarrow+\infty}\left(\mathbf{H}_{\mathrm{s}}\right)^{\mathrm{q}}=\lim _{\tau \rightarrow 0}\left(\mathbf{H}_{\mathrm{s}}\right)^{\frac{\mathrm{z}}{\tau}}=\exp \left(\mathrm{z}\left[(1-\mathrm{t}) \mathbf{D}_{1}+\mathrm{t} \mathbf{D}_{2}\right]\right)
$$

It is worth noticing that:

$$
\psi\left[\left(\mathbf{H}_{1}^{\mathrm{e}} \mathbf{H}_{2}^{\mathrm{e}}\right)^{\mathrm{q}}\right] \neq\left[\psi\left(\mathbf{H}_{1}^{\mathrm{e}} \mathbf{H}_{2}^{\mathrm{e}}\right)\right]^{\mathrm{q}}
$$

The corresponding Mueller matrix to $\mathbf{H}(z, t)$ is noted $\mathbf{M}(L-z, t)$. This means that

$$
\mathbf{M}(L-z, t)=\psi[\mathbf{H}(z, t)]
$$

with $\mathbf{M}(L, t)=\mathbf{M}_{0}$ and $\mathbf{M}(0, t)=\mathbf{I}$. Fig.2 illustrates the medium and the corresponding Mueller matrix for different values of the $z$ parameter. From the classical Mueller calculus, we have the following relation:

$$
\mathbf{M}\left(L-z_{2}, t\right) \mathbf{M}_{\mathrm{rs}}\left(z_{2}-z_{1}, t\right)=\mathbf{M}\left(L-z_{1}, t\right)
$$

where $\mathbf{M}_{\mathrm{rs}}$ stands for the Mueller matrix of the removed sandwich.

Deriving the expression of $\mathbf{M}_{\mathrm{rs}}\left(z_{2}-z_{l}, t\right)$ from Eq.(21) as Jones did [19] (for Jones matrices or Barakat [20] for the Mueller-Jones matrices), is not possible. For general Mueller matrices, the inverse of $\mathbf{M}$ does not always exist. Nevertheless, when $z_{2}=L$, Eq. (21) gives $\mathbf{M}_{\mathrm{rs}}\left(L-z_{l}, t\right)=\mathbf{M}\left(L-z_{l}, t\right)=\psi\left(\mathbf{H}\left(z_{1}, \mathrm{t}\right)\right)$.

Thus, $\psi\left(\mathbf{H}\left(z_{1}, \mathrm{t}\right)\right.$ may be also associated to a medium considered as a sandwich composed by an infinite number $q$ of elements with a thickness $\tau$. When $\tau$ approaches zero, $q \tau=z_{1}$.

In his paper on exponential versions of Mueller-Jones matrices [20], Barakat asked « whether a general Mueller matrix obeys the semigroup transformation property and thus possesses an infinitesimal generator matrix ». It is worth noticing that the approach we proposed, may be considered as a partial answer to this question for the Mueller matrices associated to a definite positive coherency matrix.

Since Eqs. (9) and (18) are similar for $\mathbf{D}_{1}=\log \left(\mathbf{H}_{1}\right), \mathbf{D}_{2}=\log \left(\mathbf{H}_{2}\right)$ and $z_{1}=1$, understanding the underlying physical meaning of Log-Euclidean interpolation process is straightforward with this latter result. The medium associated to the Mueller matrix $\mathbf{M}(t)$ is considered as a sandwich of thin laminae. Interpolating from $\mathrm{M}_{1}$ to $\mathrm{M}_{2}$ consists in changing the proportion of both the elements constituting the thin laminae. When $t$ $=0$, the thin laminae are composed by only one of the elements and by the other one when $t=1$. For all the 
in-between situations, the thin laminae are a mixture of both the elements. The parameter $t$ may be considered as a quantity homogeneous to a thickness. Fig.1-(b) illustrates this interpolating process with different values of the parameter.
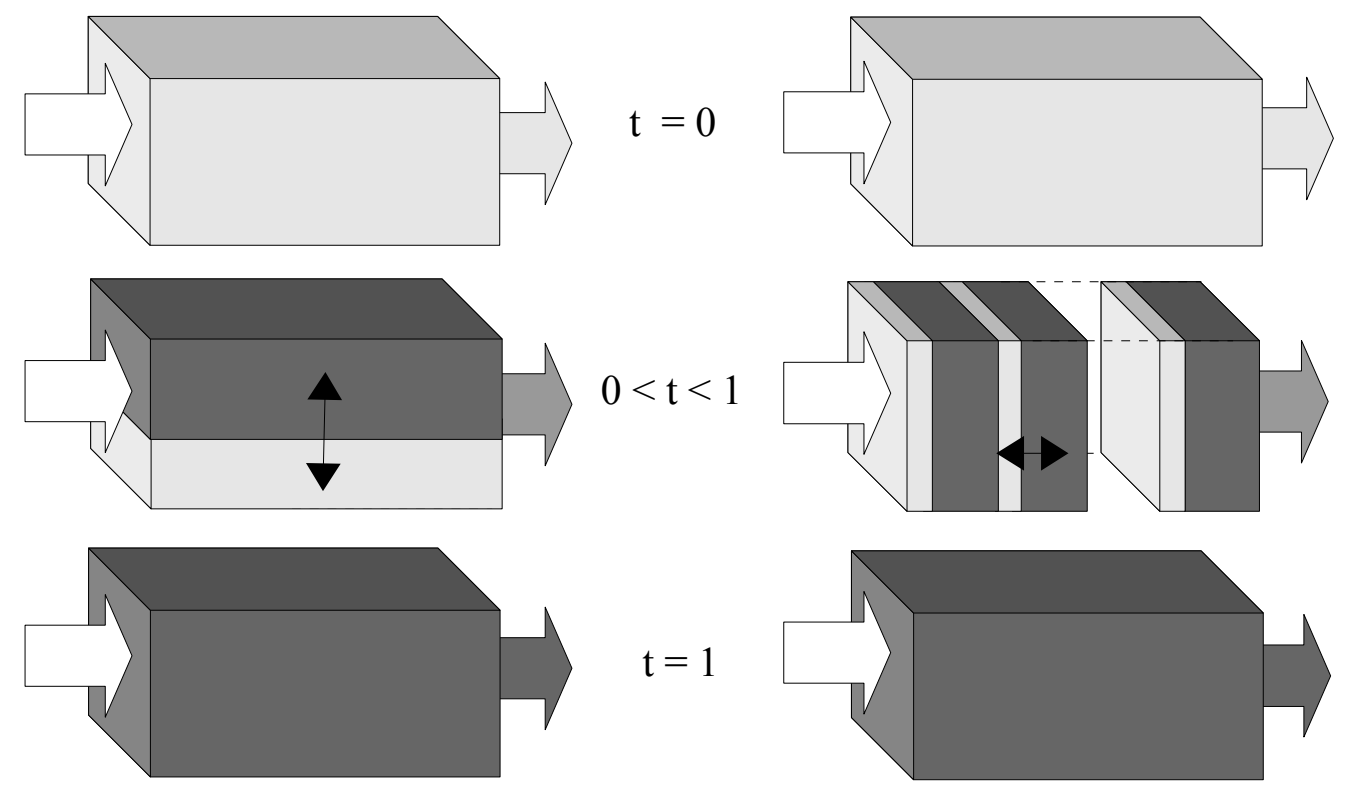

(a)

(b)

Fig.1-

Interpolation process for 3 values of the $t$ parameter. (a): Euclidean distance model. (b): Log-Euclidean distance model.

\subsection{Properties of interpolation processes}

The definition of the Hermitian matrix $\mathbf{H}$ shows that its elements are, in fact, the second-order moments of Jones matrix entries (see Eqs.(2)-(3)). Thus, $\mathbf{H}$ may be considered as the covariance matrix of the random Jones matrix entries. With a classical hypothesis of Gaussian distributions for instance, the determinant of a covariance matrix is a direct measure of the dispersion of the associated multivariate Gaussian. The reason is that the volumes of associated trust regions are proportional to the square root of this determinant [18]. For the Euclidean interpolation curve, the determinant of the interpolated HPD matrix can be strictly larger than the determinants of its end points since the induced interpolation of determinants is polynomial and not monotonic in general. Averaging two multivariate Gaussian with the same mean values can not produce a higher dispersion value. Introducing more dispersion in the interpolated Jones matrix parameters seems consequently physically acceptable only if both the multivariate Gaussian associated to the end points of the interpolated curve, have not the same average values.

For the Log-Euclidean interpolation curve, the determinant of the interpolated $H P D$ matrices is always bounded by the values of the determinants of the end points (see Appendix B for the proof of this property). Thus, the previous restriction does not applied to the Log-Euclidean interpolation. 
Consequently, when no specific information is known about the statistical characteristics of the variate related to the coherency matrix (view as a covariance matrix), the Log-euclidean interpolation process seems to be more appropriated than the Euclidean one. The choice between both these interpolation processes may thus depend on what the statistical situation is considered or what the underlying physical model is assumed.
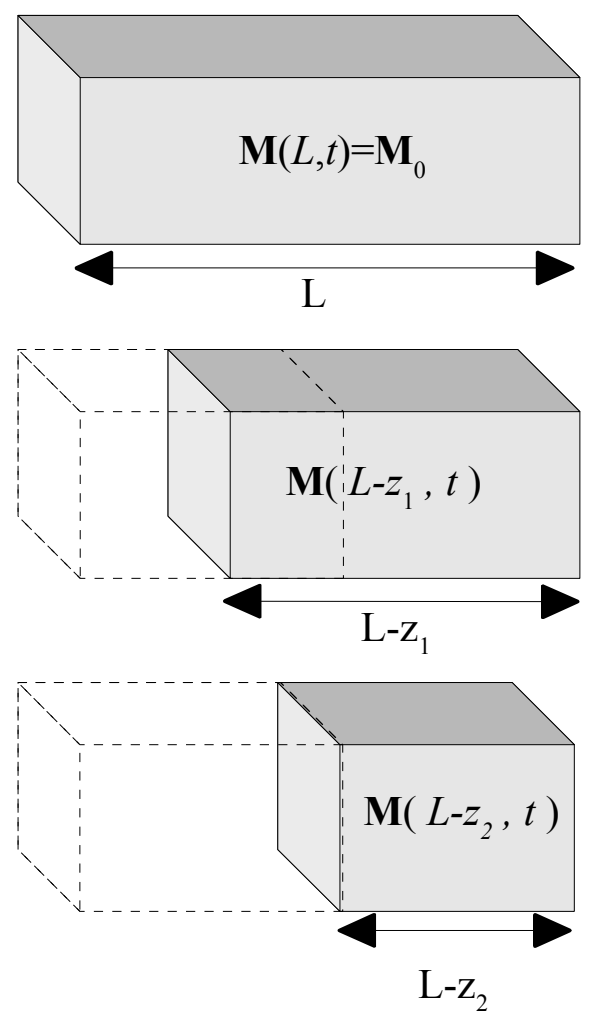

Fig.2- Medium and the different values of the $z$ corresponding Mueller matrix for parameter.

\section{4 - Conclusion}

In this work, the question of physical significance of Mueller matrix average is addressed by means of analysis of interpolation processes. We draw a comparison between a first interpolation process related to the classical Euclidean metrics framework and a second one, based on the recently introduced Log-Euclidean metrics.

This second distance may be only defined on the sub-set of positive definite coherency matrices. Since experimental coherency matrices of media exhibited in the literature are nearly always positive definite matrices, this latter hypothesis seems to be acceptable from an experimentalist point of view.

Both the associated interpolation procedures are depicted with their underlying physical model. Addressing the question of the physical meaning of Log-Euclidean process of interpolation, is founded on a very similar approach to the layered-medium interpretation proposed by Jones in the seventh paper of his series. It is worth noticing that the approach we proposed for the Mueller matrices associated to a definite positive coherency matrix, may be also considered as a partial answer to the question of the existence of a semigroup transformation property formulated by Barakat.

Based on the analysis of their respective properties, we eventually shown that the choice between both these interpolation processes may depend on what the statistical situation is considered or what the underlying physical model is assumed. 


\section{Appendix A}

It is possible to extract from $\mathbf{F}$ an Hermitian matrix $\mathbf{H}$ by doing a partial exchange of the rows of $\mathbf{F}$. This mapping $\mathbf{H}=\operatorname{Per}(\mathbf{F})$ is defined by:

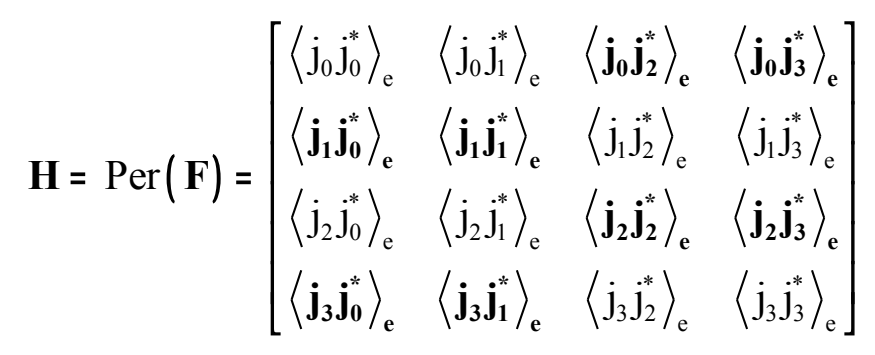

The exchanged entries of $\mathbf{F}$ (see Eq. (2)) are in bold font.

\section{Appendix B}

For a matrix $\mathbf{X}$, we have the following relation:

$$
\operatorname{det}[\exp (\mathbf{X})]=\exp [\operatorname{Tr}(\mathbf{X})]
$$

where $\operatorname{Tr}(\mathbf{X})$ and $\operatorname{det}(\mathbf{X})$ are for the trace and the determinant of $\mathbf{X}$, respectively.

From Eq.(9), we have:

$$
\begin{aligned}
\operatorname{det}[\mathbf{H}(\mathrm{t})] & =\operatorname{det}\left[\exp \left(\left[(1-\mathrm{t}) \mathbf{D}_{1}+\mathrm{t} \mathbf{D}_{2}\right]\right)\right] \\
& =\exp \left(\left[(1-\mathrm{t}) \operatorname{Tr}\left(\mathbf{D}_{1}\right)+\operatorname{trr}\left(\mathbf{D}_{2}\right)\right]\right) \\
& =\exp \left(\operatorname{Tr}\left(\mathbf{D}_{1}\right)\right)^{(1-\mathrm{t})} \exp \left(\operatorname{Tr}\left(\mathbf{D}_{2}\right)\right)^{\mathrm{t}} \\
& =(\operatorname{det}[\mathbf{H}(0)])^{(1-\mathrm{t})}(\operatorname{det}[\mathbf{H}(1)])^{\mathrm{t}}
\end{aligned}
$$

Thus, for $0 \leq t \leq 1$, we have: $\min [\operatorname{det}(\mathbf{H}(0)), \operatorname{det}(\mathbf{H}(1))] \leq \operatorname{det}(\mathbf{H}(t)) \leq \max [\operatorname{det}(\mathbf{H}(0)), \operatorname{det}(\mathbf{H}(1))]$

\section{References}

1. B. Laude-Boulesteix, A. De Martino, B. Drévillon, L. Schwartz, "Mueller Polarimetric Imaging System with Liquid Crystals", Appl. Opt. 43, 2824-2832 (2004)

2. Eric Compain, Stéphane Poirier, and Bernard Drevillon, "General and Self-Consistent Method for the Calibration of Polarization Modulators, Polarimeters, and Mueller-Matrix Ellipsometers", Appl. Opt. 38, 3490-3502 (1999)

3. A. Aiello, G. Puentes, D. Voigt, and J. P. Woerdman, "Maximum-likelihood estimation of Mueller matrices", Opt. Lett. 31, 817-819 (2006)

4. Jihad Zallat, Christian Heinrich, and Matthieu Petremand, "A Bayesian approach for polarimetric data reduction: the Mueller imaging case", Opt. Express 16, 7119-7133 (2008)

5. F Boulvert, B Boulbry, G Le Brun, B Le Jeune, S Rivet and J Cariou, "Analysis of the depolarizing properties of irradiated pig skin", J. Opt. A: Pure Appl. Opt. 7, 21-28 (2005)

6. A. Hielscher, A. Eick, J. Mourant, D. Shen, J. Freyer, and I. Bigio, "Diffuse backscattering Mueller matrices of highly scattering media", Opt. Express 1, 441-453 (1997) 
7. M. H. Smith, P. Burke, A. Lompado, E. Tanner, and L. W. Hillman, "Mueller matrix imaging polarimetry in dermatology", in Proc. SPIE 3911, 210-216 (2000)

8. Juan M. Bueno and Pablo Artal, "Double-pass imaging polarimetry in the human eye", Opt. Lett. 24, 6466 (1999)

9. M. H. Smith, E. A. Sornsin, R. A. Chipman, T. J. Tayag, "Mueller matrix imaging of GaAs/AlGaAs selfimaging beamsplitting waveguides", in Proc. Polarization Measurement, Analysis, and Remote Sensing, SPIE 3121, 47-54 (1997)

10. Lianhua Jin, Takayuki Hamada, Yukitoshi Otani, and Norihiro Umeda, Measurement of characteristics of magnetic fluid by the Mueller matrix imaging polarimeter, Opt. Eng 43, 181-185(2004), doi:10.1117/1.1631001

11. Williams M. Boothby, An introduction to Differentiable Manifolds and Riemannian Geometry-Second Edition, (Academic Press, Inc., Orlando, 1986)

12. K. Kim, L. Mandel, and E. Wolf, "Relationship between Jones and Mueller matrices for random media", J. Opt. Soc. Am. A 4, 433-437 (1987)

13. S.R. Cloude, "Group theory and polarisation algebra", Optik 75, 26-36 (1986)

14. J. J. Gil, "Characteristic properties of Mueller matrices", J. Opt. Soc. Am. A 17, 328-334 (2000)

15.A. Aiello and J. P. Woerdman, arXiv.org e-print archive math-ph/0412061 (2004)

16. J.J. Gill, E. Bernabeu, "Depolarization and polarization indices of an optical system", Opt.Acta 33, 185189 (1986)

17. J.J. Gil, J.M. Correas, P.A. Melero, C. Ferreira, Monogr.Sem. Mat. Garc' 1a de Galdeano, , (2004), availablefrom $<$ http://www.unizar.es/galdeano/actas pau/PDFVIII/pp161-167.pdf $>$

18. V. Arsigny, P. Fillard, X. Pennec, N. Ayache, "Geometric means in a novel vector space structure on symmetric positive-definite matrices", SIAM J. Matrix Anal. Appl. 29, 328-347 (2007)

19. R.C. Jones, "A new calculus for the treatement of optical systems. VII properties of the N-matrices", J. Opt. Soc. Am. 38, 671-685 (1948)

20. R. Barakat, "Exponential versions of the Jones and Mueller-Jones polarization matrices", J. Opt. Soc. Am. A 13, 158-163 (1996) 